\section{Role of emergency department observation units in the management of patients with unexplained syncope: a critical review and meta-analysis}

\author{
Filippo Numeroso', Gianluigi Mossini', Giuseppe Lippi², \\ Gianfranco Cervellin ${ }^{1}$
}

'Emergency Department, Academic Hospital of Parma, Parma, Italy

${ }^{2}$ Section of Clinical Biochemistry, University of Verona, Verona, Italy

This meta-analysis aimed to establish the role of standardized emergency department (ED) observation protocols in the management of syncopal patients as an alternative to ordinary admission. A systematic electronic literature search was performed to identify randomized controlled trials or observational studies evaluating syncopal patients managed in ED observation units. Data regarding mean length of stay, rate of etiological diagnosis, admission rate, and incidence of shortterm serious outcomes were extracted. Six mostly single-center, small sized studies characterized by high heterogeneity, were included. A total of 458 patients were included with a balanced sex distribution (male 50.2\%), a mean age of 60.1 years, and a considerable prevalence of heart disease (32.4\%). Pooled analysis of the outcomes showed a mean stay of 28.2 hours, an etiological diagnosis rate of $67.3 \%$, an admission rate of $18.5 \%$, and a very low incidence of short-term serious outcomes (2.8\%). Due to elevated diagnostic yield and low incidence of short-term adverse events, ED observation units-based management strategy seems ideal for patients with syncope. Nevertheless, further research is needed to identify criteria for selecting patients to be managed with this approach, define evaluation protocols, and confirm the safety of this strategy. Keywords Syncope; Emergency service, hospital; Observation units

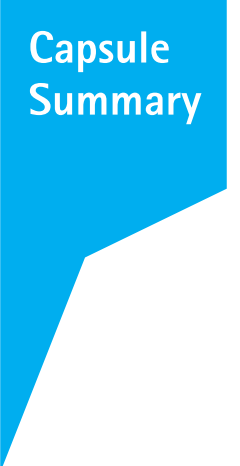

What is already known

More than half of short-term serious events after a syncope are diagnosed in the emergency department (ED); hence, consuming a large amount of resources for inpatient diagnostic workup. Therefore, there is increased interest in developing standardized observation protocols for ED patients with syncope as an alternative approach to ordinary admission.

What is new in the current study

This is the first meta-analysis aimed to evaluate the role of brief ED observation protocols in the management of patients with undetermined syncope.
elSSN: 2383-4625

Received: 20 April 2017

Revised: 8 May 2017

Accepted: 8 May 2017

Correspondence to: Filippo Numeroso Emergency Department, Academic Hospital of Parma, via Gramsci 14, 43126 Parma, Italy E-mail: fnumeroso@gmail.com

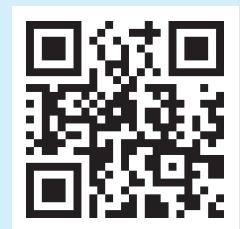

How to cite this article:

Numeroso F, Mossini G, Lippi G, Cervellin G. Role of emergency department observation units in the management of patients with unexplained syncope: a critical review and meta-analysis. Clin Exp Emerg Med 2017;4(4):201-207.

This is an Open Access article distributed under the terms of the Creative Commons Attribution Non-Commercial License (http:// creativecommons.org/licenses/by-nc/4.0/). 


\section{INTRODUCTION}

Syncope is a daily challenge for emergency physicians (EPs) since it represents approximately $3 \%$ of the emergency department (ED) visits, $6 \%$ of hospital admissions, ${ }^{1}$ and is also responsible for large hospital expenses (i.e., the total annual cost is estimated at 2.4 billion dollars in the US). ${ }^{2}$ The evaluation of patients with syncope in the ED is challenging as it is multifaceted, and often vague in nature since most patients are asymptomatic upon arrival; therefore, the diagnosis can only be done presumptively. The potential causes of syncope are broad, ranging from benign etiologies to life-threatening cardiovascular conditions. Due to the risk of sudden death, many EPs often chose a 'safe' management method for these patients by admitting them to the hospital.

Although the diffusion of guidelines into practice has allowed a considerable improvement in the clinical management of patients with syncope during the last decades, ${ }^{3}$ the admission rate remains high (38\% to 42\%) compared to the incidence of shortterm events. ${ }^{4}$ According to the Position Paper of the Canadian Cardiovascular Society, ${ }_{1}^{5}$ the percentage of short-term (i.e., within 1 month) non-fatal severe outcomes is approximately $12 \%$ with low incidence of deaths (0.7\%) throughout the same period. It has also been reported that more than half of these events $(7.5 \%$, i.e., $62.5 \%$ of the total number of events) occur at the time of $E D$ admission. Therefore, it seems reasonable that only a minority of patients will benefit from further assessment and treatment after a thorough evaluation in the ED. Patients with a negative workup in the ED do not benefit from hospital admission since the risk of a life-threatening event is extremely low even in the presence of cardiovascular risk factors. ${ }^{4,6}$

On the other hand, it is still uncertain whether hospitalization may help reduce adverse events in patients with unexplained syncope. The decision to admit a patient should also take into consideration many variables such as incremental costs, the risk of adverse events during hospital stay and the actual effectiveness of hospitalization for the clinical management. ${ }^{7}$

Due to the high prevalence of syncope, the multiple etiologies and the incremental expenditures emerging from inpatient diagnostic workup, there is increasing interest in ED-based observation protocols as an alternative approach to ordinary admission. According to these standardized observation protocols, patients with unexplained syncope can be observed and monitored in an appropriate area of the ED, conventionally known as ED observation unit (EDOU). This approach makes it possible to optimize the diagnostic process, allowing tailoring the use of hospitalization and diagnostic investigations to the individuals. According to the first international workshop on risk stratification for syncopal pa- tient in the ED7 held in Gargnano (Italy) in 2013, EDOUs admission was considered particularly suitable for high- and intermediate-risk patients since it would help in deciding between patient admission or discharge, eventually followed by a fast-track path to an outpatient syncope unit.

To the best of our knowledge, no meta-analyses have been published so far with the aim of evaluating the role of brief EDOU protocols in the management of patients with undetermined syncope. Therefore, we performed a critical review of the literature to summarize the available evidence on syncope management in the ED.

\section{METHODS}

\section{Search strategy and study selection}

A systematic electronic search was performed in Medline (via PubMed), Embase, clinicaltrials.gov and Cochrane Library for identifying randomized controlled trials (RCTs) and observational studies using the search keywords "syncope," "emergency department," "observation," "unit," "protocol(s)." These terms were combined using Boolean operators "AND" and "OR," with no date restriction. The references of the retrieved articles were also scrutinized to identify additional and pertinent items.

Two independent reviewers (FN and GM) initially screened the title and/or abstract of all articles for possible inclusion. When potentially eligible, the complete article was then scrutinized according to the following criteria: (1) studies investigating syncopal patients managed in a short-stay observation unit located in the $E D$, and (2) reporting at least one of the following: mean length of stay in the EDOU, rate of admission, rate of etiological diagnosis, incidence of short-term serious events. Exclusion criteria were (1) duplicate reporting (in which case the manuscript reporting the largest sample of patients was selected), (2) differentiated syncope patients (i.e., studies recruiting patients with an already defined diagnosis of syncope, e.g., cardiovascular cause only), (3) studies enrolling patients with near syncope, or (4) articles written in languages other than English.

\section{Data extraction}

Two independent reviewers (FN and GM) retrieved the following information on pre-specified data collection forms: authors, journal, year of publication, location, study design, enrollment criteria, number and demographic characteristics, prevalence of heart diseases (i.e., coronary heart diseases, heart failure, or arrhythmias), mean length of stay in the EDOU, rate of admission, rate of etiological diagnosis, and incidence of short-term adverse outcomes. Authors were contacted if the data needed clarification or were incomplete. Additional information was included when the re- 
sponse was obtained; otherwise, the available data were used for our analyses. Only short-term adverse events were considered for evaluating the safety of EDOU model with the assumption that only these outcomes could influence the decision making about the patient's disposition. Notably, it was already demonstrated that long-term prognosis is substantially unaffected by hospital admission and is related to comorbidity. ${ }^{8}$

\section{Assessment of the quality of studies}

The methodological quality of the studies included was assessed by two reviewers (GL and GC) independently, using the Jadad score ${ }^{9}$ for RCTs and the Newcastle Ottawa Scale ${ }^{10}$ for observational, non-randomized studies.

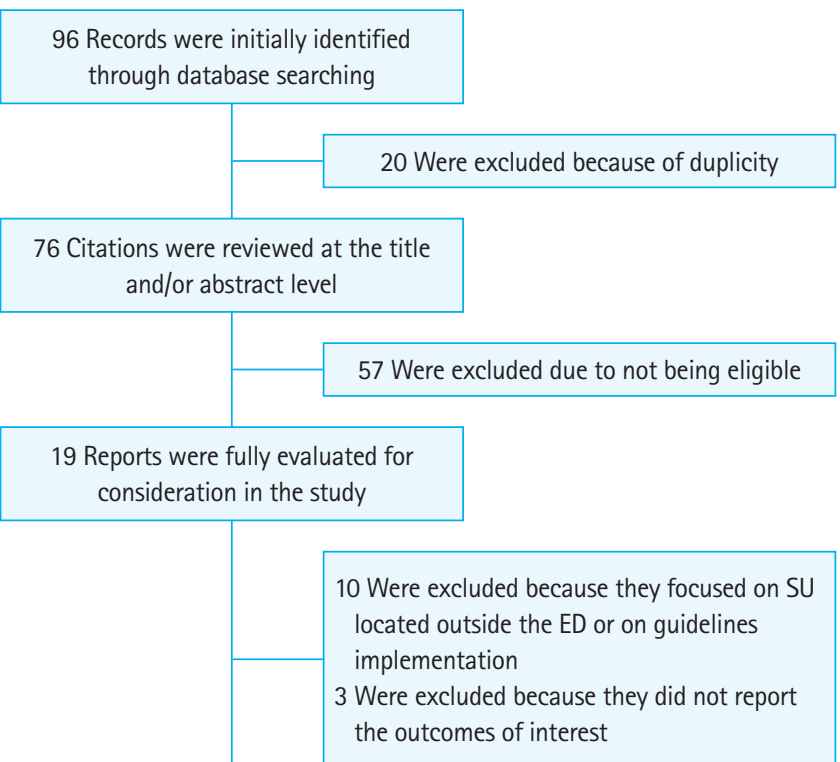

6 Reports were finally included in the meta-analysis

Fig. 1. Flowchart of the literature search and study selection procedure. SU, syncope unit; ED, emergency department.

\section{Data analysis}

Pooled estimates for the outcomes of interest were analyzed using random effects meta-analysis. Admission rate, etiological diagnosis, and short-term adverse events were reported as proportions and 95\% confidence intervals. With regard to the specified outcomes, heterogeneity was assessed using the $l^{2}$ statistic where thresholds of 25\%,50\%, and 75\% indicated low, moderate and high heterogeneity, respectively. The mean length of stay in the EDOU (continuous variable) was reported as weighted mean with a pooled standard deviation as a measurement of uncertainty. Statistical analyses were performed with MedCalc Statistical Software ver. 16.8.4 (MedCalc Software, Ostend, Belgium).

\section{RESULTS}

\section{Study selection}

Fig. 1 shows the flowchart for the literature search strategy and the study selection procedure. Initially, 96 records were identified during the database search. After the exclusion of duplicates, 76 citations were reviewed at the title and/or abstract level. A total number of 57 documents were excluded because they were review articles, case reports, or studies enrolling EDOU cohorts of patients to evaluate for other issues instead of evaluating syncope only. Nineteen reports were then included for further analysis. Ten items were excluded since they were based on syncope facilities located outside the ED or focused on guidelines implementation in the ED, whereas three studies were excluded because they did not report the outcomes of interest. Therefore, six studies were included in our meta-analysis. ${ }^{11-16}$

\section{Study characteristics}

The main characteristics of the six studies are shown in Table 1. ${ }^{11-16}$ Four were observational studies and two were RCTs. These were mostly single-center studies, conducted in 2004 to 2016, three each in the United States and Europe (two in Italy and one in Spain). The sample size of these studies ranged between 27 and

Table 1. Main characteristics of the studies included in the meta-analysis

\begin{tabular}{|c|c|c|c|c|c|c|c|c|}
\hline Study & Year & Country & Design & $\begin{array}{l}\text { Patients } \\
\text { (n) }\end{array}$ & $\begin{array}{l}\text { Male } \\
(\%)\end{array}$ & $\begin{array}{c}\text { Mean age } \\
(y r)\end{array}$ & $\begin{array}{c}\text { CV disease } \\
(\%)\end{array}$ & Inclusion criteria \\
\hline Shen et al. ${ }^{11}$ & 2004 & US & RCT & 51 & 49 & 64 & 43 & Patients with undetermined syncope at intermediate risk \\
\hline Rodriguez-Entem et al. ${ }^{12}$ & 2008 & Spain & OS & 199 & 54 & 67 & NA & Patients not selected on a risk category basis \\
\hline Sun et al. ${ }^{13}$ & 2013 & US & $\mathrm{RCT}$ & 62 & 47 & 65 & 23 & Patients with undetermined syncope at intermediate risk \\
\hline Grossman et al. ${ }^{14}$ & 2015 & US & OS & 27 & 33 & 53 & 22 & Patients not selected on a risk category basis \\
\hline Ungar et al. ${ }^{15}$ & 2016 & Italy & OS & 60 & 40 & 68.5 & 55 & Patients not selected on a risk category basis \\
\hline Numeroso et al. ${ }^{16}$ & 2016 & Italy & OS & 59 & 59 & 66.7 & 15 & Patients with undetermined syncope at intermediate risk \\
\hline
\end{tabular}

$\mathrm{CV}$, cardiovascular; RCT, randomized controlled trial; OS, observational study; NA, not applicable. 
199, with a balanced distribution for sex and with ages of 53 to 68.5 years. The prevalence of cardiovascular diseases ranged between 15\% and 55\%. A total of 458 patients were included in these studies, with a balanced sex distribution (men were $50.2 \%$ of total); the overall mean age of the patient was relatively young, (60.1 years) albeit, with considerable prevalence of heart disease (32.4\%).

Enrollment criteria in these studies varied; three studies were focused on patients at intermediate risk (with slight differences in identification criteria), while the remaining studies included patients regardless of their risk category, only excluding those with acute disease already present at ED admission or a non-syncopal transient loss of consciousness (Supplementary Table 1). ${ }^{11-16}$

\section{Quality assessment}

According to Jadad scores, one RCT was high quality and one RCT was low quality (Supplementary Table 2). ${ }^{11,13}$ According to the Newcastle-Ottawa Scale, the two observational studies were high quality and one observational study was moderate quality; for the last one, the Newcastle-Ottawa Scale was only partially applicable, so the total sum was not considered (Supplementary Table 3). ${ }^{12,14-16}$

\section{Study results}

Table 2 shows the endpoints reported by the studies included in this meta-analysis. ${ }^{11-16}$ The SEEDS Study, ${ }^{11}$ the first study published, was a prospective, single-center RCT investigating the usefulness of a standardized pathway for the evaluation and management of patients presenting to the ED with syncope. It was based on a designated ED syncope unit (EDSU) equipped with multidisciplinary diagnostic resources for assessment of syncopal patients at intermediate risk of cardiovascular outcomes. The study showed that the model based on EDSU significantly improved the rate of etiological diagnosis (10\% in standard care patients versus $67 \%$ in those evaluated in EDSU), and was effective in lowering both hospital admission rate (43\% in the group with patients evaluat-

Table 2. Endpoints reported by the studies included

\begin{tabular}{|c|c|c|c|c|c|}
\hline Study & Patients & $\begin{array}{l}\text { Mean LOS } \\
\text { hours (SD) }\end{array}$ & $\begin{array}{l}\text { Admission } \\
\text { rate }\end{array}$ & $\begin{array}{l}\text { Etiological } \\
\text { diagnosis }\end{array}$ & $\begin{array}{c}\text { Short term } \\
\text { outcomes }\end{array}$ \\
\hline Shen et al. ${ }^{11}$ & 51 & NA & $22(43.1)$ & 34 (66.6) & NA \\
\hline Rodriguez-Entem et al. ${ }^{12}$ & 199 & $19(15)$ & $20(10.0)$ & $131(65.8)$ & NA \\
\hline Sun et al..$^{13}$ & 62 & $29(15)$ & $9(14.5)$ & NA & $2(3.2)$ \\
\hline Grossman et al. ${ }^{14}$ & 27 & NA & NA & $12(44.4)$ & NA \\
\hline Ungar et al. ${ }^{15}$ & 60 & $34(8)$ & 7 (11.6) & $40(66.6)$ & $3(5.0)$ \\
\hline Numeroso et al. ${ }^{16}$ & 59 & 41 (17) & NA & $42(71.2)$ & $0(0.0)$ \\
\hline
\end{tabular}

Values are presented as number (\%) unless otherwise indicated. LOS, length of stay; SD, standard deviation; NA, not applicable. ed in EDSU vs. $98 \%$ in the standard care group) and the total length of hospital stay in the cases of ordinary hospitalization (total patient-hospital days reduced from 140 with standard care to 64 in the EDSU group). Notably, hospital admissions were mostly related to therapeutic purposes (19/22, i.e., 86\%) rather than caused by the absence of a diagnosis at the end of an EDSU evaluation. During the long-term follow-up, no significant differences were found between the EDSU and standard-care group, both in actuarial survival (97\% and 90\%, $\mathrm{P}=0.30)$ and survival from recurrent syncope ( $88 \%$ and $89 \%, \mathrm{P}=0.72$ ).

In an ensuing study, Rodriguez-Entem et al. ${ }^{12}$ investigated the effectiveness of a two-phased protocol based on a strict application of the European Society of Cardiology guidelines and on the availability of a dedicated area of the ED where patients could be monitored until they were discharged or admitted. Phase 1 was based on initial clinical assessment with electrocardiogram and monitoring, whereas phase 2 entailed further evaluation using inpatient electrophysiological study or outpatient tilt table test building on the findings of the electrocardiogram and echocardiogram. This approach facilitated higher rates of etiological diagnosis (65.8\% upon discharge from the ED, 78\% after completion of the diagnostic protocol), with a mean stay of 19 hours in the ED and low rates of hospital admissions (10\%).

More recently, the Emergency Department Observation Syncope Protocol study ${ }^{13}$ showed that an ED observation syncope protocol for intermediate-risk patients was effective in facilitating lower hospital admission rate (15\% vs. 92\%), shorter mean hospital length of stay (29 vs. 47 hours), and lower hospital costs (mean cost at index visit 1,400 vs. 2,420 US dollars), with no difference in the rate of short-term serious outcomes after hospital discharge (3\% among patients managed with the ED observation protocol) compared to the patients admitted.

In the study by Grossman et al., ${ }^{14}$ a cohort of ED syncopal patients were retrospectively reviewed. The diagnostic yield of the three management modalities based on EPs judgment in different hospital settings were compared; it showed that patients managed with full hospitalization ( $>1$ day) were more likely to be discharged with a definite etiology of syncope (74\%) as compared to those managed with a 1-day admission to an inpatient ward (64\%) or evaluated in the EDOU (44\%). Nevertheless, this data may be biased because serious causes of syncope (e.g., pulmonary embolism, sepsis, ventricular dysrhythmias, stroke, or acute myocardial infarction) were identified among a considerable number of fully admitted patients (38\%). Therefore, these patients should be considered as having syncope associated with an acute disease already present at ED admission rather than undetermined syncope complicated by a short-term adverse outcome. Both 
1-day admission and EDOU patients received a lower percentage of definite etiologic diagnoses, $36.3 \%$ and $56.6 \%$, respectively; but no information was provided about the tests needed to reach a diagnosis during such a brief hospital stay ( $\leq 24$ hours), except for low number of echocardiograms.

Ungar et al. ${ }^{15}$ investigated the impact of a structured hospital organization based on the presence of a short-stay observation unit inside the ED and an outpatient syncope unit on the admission rate and the clinical outcomes, such as death and re-admission. The availability of these facilities significantly improved the diagnostic performance, without increasing short-term adverse events. More specifically, patients managed in short-stay observation units had relatively short stays. Eighty percent of them were kept in for $\leq 24$ hours while the remaining 20\% stayed for 24 to 48 hours; the mean length of stay was 34 hours with a standard deviation of 8 . When compared with those managed by ordinary admission, these patients had a lower rate of unexplained syncope (33.4\% vs. $51.8 \%$, respectively), and a low incidence of short-term adverse events ( 3 re-admissions for non-fatal-events, corresponding to $5 \%$ of the total cohort). In the IRiS Study, ${ }_{1}^{16}$ the management of patients with undetermined syncope at intermediate risk was also analyzed, showing that a short hospital stay in the EDOU yielded a better diagnostic efficiency (unexplained cases, $28.8 \%$ vs. $51 \%$ ), and significantly lower costs (mean expenditure for each case, 431.80 vs. 2,969 US dollars) compared to the ordinary hospital admission, and this was mostly attributable to shorter length of stay (mean length of stay, $41 \mathrm{vs.}$ 211 hours). Nevertheless, these data were partially biased because EDOU patients were younger with fewer comorbidities and heart diseases, and cardiac monitoring devices are more readily available in the EDOU as compared to the ordinary wards.

\section{Pooled estimates for the outcomes}

Table 3 shows the pooled estimates for the outcomes considered. The mean length of stay was 28.2 hours (95\% confidence interval, 26.7 to 29.7). An etiological diagnosis could be reached in approximately two-thirds of the patients (67.3\%) while overall admission rate was low (18.5\%), and short-term adverse outcomes were seen in a very small percentage of patients (2.8\%). Notably,

Table 3. Pooled estimates for the outcomes

\begin{tabular}{lcc}
\hline Outcome & Sample size & Pooled estimates, \% (95\% Cl) \\
\hline Mean length of stay in the EDOU & 380 & $28.2(26.7-29.7)$ \\
Etiological diagnosis & 396 & $67.3(58.1-75.9)$ \\
Admission rate & 372 & $18.5(7.8-32.4)$ \\
Serious outcomes & 181 & $2.8(0.4-7.2)$ \\
\hline
\end{tabular}

$\mathrm{Cl}$, confidence interval; EDOU, emergency department observation unit. heterogeneity was moderate for the rate of etiological diagnosis (I-squared, 31.3\%) and high for both admission rate (I-squared, 88.4\%) and the rate of short-term negative outcomes (I-squared, $51.4 \%$ ). Similarly, the coefficient of variation of the length of stay was found to be rather high (approximately 50\%), indicating a high dispersion around the mean.

\section{DISCUSSION}

\section{Summary of evidence}

The results of this meta-analysis show that the available studies on the role of the EDOUs in the management of patients with syncope are scarce and small-sized, mainly based on a single center, and they present significant differences in methodology. Therefore, additional research on this important issue is recommended to better understand the unresolved questions.

The main gap concerns the lack of definite criteria for identifying patients with indeterminate syncope, for whom evaluation in the EDOU may be appropriate. Notably, the mean age of the entire study population included in this meta-analysis is relatively low (60 years), and this is probably attributable to the fact that patient enrolment, especially in observational studies, was dependent on the individual judgement of the EPs, who usually tend to manage younger and healthier patients with short observation or discharge.

The EDOSP and IRiS studies confirmed that patients at intermediate risk, namely patients of any age with stable heart disease or comorbidities, could be reliably managed with intensive clinical monitoring in the EDOU rather than with hospital admission. Conversely, these patients tend to be hospitalized, thus leading to prolonged stays and higher costs with no improvements in diagnostic yield. ${ }^{16}$

A second important drawback is related to the lack of consensus on the equipment and tests necessary for the rigorous evaluation of patients with syncope in the EDOU. This is mirrored by the variability of instrumental investigations and management practices among the different studies. Hence, standardized EDOU protocols should be defined to harmonize clinical and instrumental procedures, and optimizing the ED approach to syncopal patients. According to the national regulations in Italy, the length of stay in the EDOUs should be no longer than 36 hours. ${ }^{17}$ Therefore, the short length of stay estimated in this meta-analysis (28.2 hours) further supports the reliability of managing syncope in the EDOU. The adoption of a standardized-care pathway will predictably lead to optimization of the diagnostic yield, as has been shown in the EGSYS-2 Study. ${ }^{18}$

An additional gap in knowledge is the actual safety of the EDOU 
model, since only a small number of studies have analyzed the short-term prognosis of patients discharged after being observed during a short stay. Since protection against adverse events ${ }^{8}$ is the main purpose of admitting patients with undetermined syncope, the preliminary results obtained from the studies available must be confirmed with large, multicenter trials.

The admissions were overall infrequent (18.5\%), but this result is biased due to high level of inter-study heterogeneity (I-square $88.4 \%$ ), with the ordinary hospitalization rate being as high as $43 \% .{ }^{11}$ Although this finding is probably influenced by the lack of a standardized diagnostic protocol, the value of the EDOU in the management of patients with syncope is also related to the possibility of performing a more accurate risk stratification to identify patients needing admission because of being at high risk.

\section{Limitations}

This study has two main limitations. First, inclusion criteria were different among the studies; therefore, reporting average results might be misleading. Second, the small number of the studies included limits the usefulness of the pooled estimates as compared to the results of individual investigations.

\section{CONCLUSION}

The considerable increase in ED overcrowding, combined with lower availability of inpatient hospital beds, represent critical social and health care issues. Although syncope appears to be an ideal condition for an EDOU-based management strategy, additional research is needed to, (1) define protocols for patient management, (2) identify criteria for the appropriate selection of syncopal patients who may be managed according to this pathway, and (3) confirm the safety of this model, based on the absence of short term serious events in syncopal patients with a negative ED evaluation.

\section{SUPPLEMENTARY MATERIALS}

Supplementary Tables are available from: https://doi.org/10.15441/ ceem.17.231

Supplementary Table 1. Enrollment criteria of the included studies in the meta-analysis

Supplementary Table 2. Quality assessment of randomized controlled trials

Supplementary Table 3. Quality assessment of observational studies

\section{CONFLICT OF INTEREST}

No potential conflict of interest relevant to this article was reported.

\section{REFERENCES}

1. Manolis AS, Linzer M, Salem D, Estes NA 3rd. Syncope: current diagnostic evaluation and management. Ann Intern Med 1990;112:850-63.

2. Task Force for the Diagnosis and Management of Syncope; European Society of Cardiology (ESC); European Heart Rhythm Association (EHRA), et al. Guidelines for the diagnosis and management of syncope (version 2009). Eur Heart J 2009;30: 2631-71.

3. Brignole $M$, Menozzi $C$, Bartoletti $A$, et al. A new management of syncope: prospective systematic guideline-based evaluation of patients referred urgently to general hospitals. Eur Heart J 2006;27:76-82.

4. Brignole $M$, Hamdan MH. New concepts in the assessment of syncope. J Am Coll Cardiol 2012;59:1583-91.

5. Sheldon RS, Morillo CA, Krahn AD, et al. Standardized approaches to the investigation of syncope: Canadian Cardiovascular Society position paper. Can J Cardiol 2011;27:246-53.

6. Morag RM, Murdock LF, Khan ZA, Heller MJ, Brenner BE. Do patients with a negative Emergency Department evaluation for syncope require hospital admission? J Emerg Med 2004; 27:339-43.

7. Costantino G, Sun BC, Barbic $F$, et al. Syncope clinical management in the emergency department: a consensus from the first international workshop on syncope risk stratification in the emergency department. Eur Heart J 2016;37:1493-8.

8. Costantino G, Perego F, Dipaola F, et al. Short- and long-term prognosis of syncope, risk factors, and role of hospital admission: results from the STePS (Short-Term Prognosis of Syncope) study. J Am Coll Cardiol 2008;51:276-83.

9. Jadad AR, Moore RA, Carroll D, et al. Assessing the quality of reports of randomized clinical trials: is blinding necessary? Control Clin Trials 1996;17:1-12.

10. Wells GA, Shea B, O'Connell D, et al. The Newcastle-Ottawa Scale (NOS) for assessing the quality of nonrandomised studies in meta-analyses [Internet]. Ottawa, ON: Ottawa Hospital Research Institute [cited 2017 Jun 1]. Available from: http:// www.ohri.ca/programs/clinical_epidemiology/oxford.asp.

11. Shen WK, Decker WW, Smars PA, et al. Syncope Evaluation in the Emergency Department Study (SEEDS): a multidisciplinary approach to syncope management. Circulation 2004;110:363645.

12. Rodriguez-Entem F, Gonzalez-Enriquez S, Olalla-Antolin JJ, et al. Management of syncope in the emergency department without hospital admission: usefulness of an arrhythmia unit coordinated protocol. Rev Esp Cardiol 2008;61:22-8. 


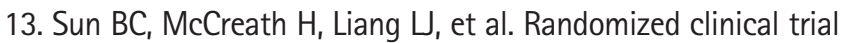
of an emergency department observation syncope protocol versus routine inpatient admission. Ann Emerg Med 2014;64: 167-75.

14. Grossman AM, Volz KA, Shapiro NI, et al. Comparison of 1-day emergency department observation and inpatient ward for 1-day admissions in syncope patients. J Emerg Med 2016;50: 217-22.

15. Ungar A, Tesi F, Chisciotti VM, et al. Assessment of a structured management pathway for patients referred to the Emergency Department for syncope: results in a tertiary hospital. Europace 2016;18:457-62.

16. Numeroso F, Mossini G, Giovanelli M, Lippi G, Cervellin G. Short- term prognosis and current management of syncopal patients at intermediate risk: results from the IRiS (Intermediate-Risk Syncope) study. Acad Emerg Med 2016;23:941-8.

17. Italian Ministry on Health, General Direction of Health Planning. Intensive short observation, national guidelines [Internet]. [place unknown]: Italian Ministry on Health; 2001 [cited 2017 Jun 1]. Available from: http://www.aliceitalia.org/cms_ bko_elfinder_1_2/files/TRIAGE.pdf.

18. Brignole $M$, Ungar A, Bartoletti $A$, et al. Standardized-care pathway vs. usual management of syncope patients presenting as emergencies at general hospitals. Europace 2006;8: 644-50. 